\section{Constant Radiation Characteristics for Log-Periodic Dipole Array Antennas}

\author{
Robert Lehmensiek and Dirk I. L. de Villiers
}

\begin{abstract}
Given the correct terminal voltages on each element of a log-periodic dipole array constant radiation characteristics can be achieved for an omni-directional radiation pattern. In this communication it is shown that this can only be achieved through optimization, correcting a previously reported oversimplification which resulted in an analytical approach being tractable. This oversimplification assumed that vanishing terminal currents on a dipole's terminal/feed element implies no radiation from that dipole. This is clearly non-physical because, zeroing the terminal current on a dipole's feed element is the same as disconnecting its two arms, while having no radiation from that dipole implies that in effect both arms be entirely removed. We show that the effect of currents induced in the disconnected arms of a dipole cannot in general be ignored.
\end{abstract}

Index Terms-Antenna feeds, Antenna radiation patterns, Broadband communication, Dipole antennas, Log periodic antennas.

\section{INTRODUCTION}

For log-periodic dipole arrays (LPDAs) [1], as shown for example in Fig. 1 for the two dipole case, an equivalent circuit model representing the currents and active input impedances at the antenna ports for each dipole in the array is given in Fig. 2. The circuit model for dipole $i$ consists of $V_{i}$ the excitation voltage source on dipole $i, Z_{g i}$ the exciter's internal impedance, $Z_{i i}$ the dipole's input impedance and $V_{c i}$ the coupled voltage from the other dipoles in the array,

$$
V_{c i}\left(f_{i}\right)=\sum_{k=1, k \neq i}^{N} Z_{i k}\left(f_{i}\right) I_{k}\left(f_{i}\right),
$$

where $Z_{i k}$ is the mutual impedance between dipoles $i$ and $k$ and $I_{k}$ is the actual terminal current of dipole $k$ at frequency $f_{i}$ [2]. For a LPDA the lengths (and spacings) of the dipoles increase logarithmically from the shortest dipole resulting in dipoles that may be electrically significantly longer (or shorter) than a half-wavelength at a frequency within the bandwidth of the LPDA, which is per definition broadband. Thus, several characteristic current modes can exist on the electrically larger dipoles. For an array with equal half-wavelength dipoles this is not the case, and only the fundamental current mode, as given by the thin wire approximation model in, for instance, [2] needs to be considered. The above circuit model in (1) is valid irrespective of the number of modes that exist on a particular dipole.

Recently, a paper on the conditions for a LPDA to have

Manuscript received November 30, 2013. This work was financially supported by the Department of Science and Technology (DST) of South Africa.

R. Lehmensiek is with EMSS Antennas (Pty) Ltd., Stellenbosch, 7600, South Africa (e-mail: lehmensk@ieee.org).

D. I. L. de Villiers is with the Department of Electrical and Electronic Engineering, Stellenbosch University, Stellenbosch, 7600, South Africa (e-mail: ddv@sun.ac.za). constant radiation characteristics (at least at the radiating element's resonance frequency) was presented [3]. According to [3], constant radiation is achieved if the radiation of the array is purely due to the radiation of a single dipole that is at resonance and all the other dipoles do not contribute to the radiation. It is thus implied that the radiation pattern of the resonant dipole in the array is the same as that which would be obtained by the same dipole in free space. In order to achieve single dipole radiation, it is postulated in [3] that all the terminal currents need to be zero apart from the one of the resonant dipole, i.e. $I_{k}\left(f_{i}\right)=0$, for $k=1, \ldots, \mathrm{N}, k \neq i$. Given this condition the voltage excitations, $V_{k}$, for the non-resonant dipoles can easily be derived, as was done in [3] (albeit with a number of typographical errors), and are:

$$
V_{k}\left(f_{i}\right)=\frac{Z_{k i}\left(f_{i}\right)}{Z_{g i}\left(f_{i}\right)+Z_{i i}\left(f_{i}\right)} V_{i}\left(f_{i}\right) .
$$

This model is correct for the case that only the fundamental current mode exists on all of the dipoles of the array. In the case of an LPDA this is an oversimplification as higher order modes do exist due to the varying dipole lengths and mutual coupling between dipoles.

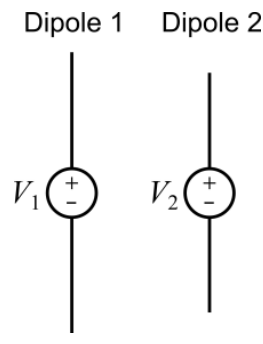

Fig. 1. A two-element log-periodic dipole array.

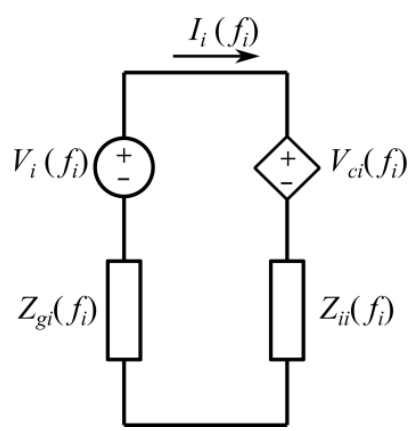

Fig. 2. Circuit model representation for Dipole $i$ of a general log-periodic dipole array.

Although an equivalent circuit model representation of an antenna element represents a good model for the terminal impedance of that element, equivalent circuit models do not model the currents away from the terminal ports and therefore cannot in general be used to predict the radiation characteristics of the antenna. The method suggested in [3] indeed only forces open circuits at the center of all the non-resonant dipoles, and does not remove these elements from the problem. Fields from the resonant or driven element will still scatter from these extra, open circuited, elements and alter the radiation pattern. Thus, a condition for constant radiation cannot be determined purely 
from the circuit models representing the terminal impedances.

In this communication we will show in Section II through a detailed parametric study of a two-element LPDA that the condition given in [3] will in general not lead to constant radiation of a log-periodic array. It will be shown that constant radiation can be achieved, but the array excitations cannot be determined analytically and have to be determined numerically, typically by brute force optimization. In Section III a five-element log-periodic dipole array is analyzed. Section IV is the conclusion.

\section{Two-Element LoG-PERIODIC DIPOLE ARRAY}

A two-element LPDA is shown in Fig. 1, where Dipole 2 is scaled from Dipole 1 with a scaling factor of $\tau$. Dipole 1 is resonant at frequency $f_{1}$ and Dipole 2 at $f_{2}=f_{1} / \tau$. The geometrical dimensions are as follows: length of Dipole 1 is $68.63 \mathrm{~mm}$, the spacing between the dipoles is $6.242 \mathrm{~mm}$, and the scaling factor $\tau=1 / 1.18$. These are the same dimensions as used in [3]. The frequency $f_{2}=3.04 \mathrm{GHz}$. The dipoles were modeled as thin wires with radii of $0.1 \mathrm{~mm}$. Similar results are found when the dipoles are modeled as thin strips, as was done in [3].

A detailed parametric study can be performed on the two-element array model using the commercially available code FEKO [4]. The parameters that can be varied are the amplitude and phase of the voltage source driving the one dipole with the other dipole excited with $1 \angle 0^{\circ} \mathrm{V}$.

Firstly we investigate the radiation characteristics of the array at the lower frequency $f_{1}=2.5763 \mathrm{GHz}$. This may be achieved by either evaluating the full array and complex excitations in the method of moments model, or by considering the embedded element patterns and calculating the total pattern as a weighted sum of the individual embedded patterns. Dipole 1 is excited with a $1 \angle 0^{\circ} \mathrm{V}$ voltage source and the amplitude of the voltage source on Dipole 2 is varied from $0 \mathrm{~V}$ to $1 \mathrm{~V}$ and the phase from $0^{\circ}$ to $360^{\circ}$. Both the radiation characteristics of the array and the current distribution on Dipole 2 were examined. Fig. 3(a) gives the maximum of the magnitude of the current that is distributed along the length of Dipole 2, i.e. the combination of the current due to coupling from Dipole 1 and that due to the excitation voltage source $V_{2}$. Fig. 3(b) shows the maximum gain deviation from the peak gain in the H-plane. The objective according to [3] is to get an array radiation pattern that is omni-directional and equal to that of a free space dipole. The minima of the contour plots are summarized in Table I, together with the voltage obtained from (2), and are superimposed on the contour plots of Fig. 3. At these minima the magnitude of the current distribution along the length of the dipoles and the resultant H-plane radiation pattern of the array are shown in Fig. 4(a) and Fig. 4(b) respectively. In Fig. 4(b) the $-90^{\circ}$ and $+90^{\circ}$ angles are in the plane of the dipole array and the direction from the terminal of Dipole 1 to the terminal of Dipole 2 is towards the $+90^{\circ}$ angle. The current distribution on the dipoles and the resultant gain pattern of the array for the case that Dipole 2 is short circuited, i.e. $V_{2}=0 \mathrm{~V}$, are also shown.

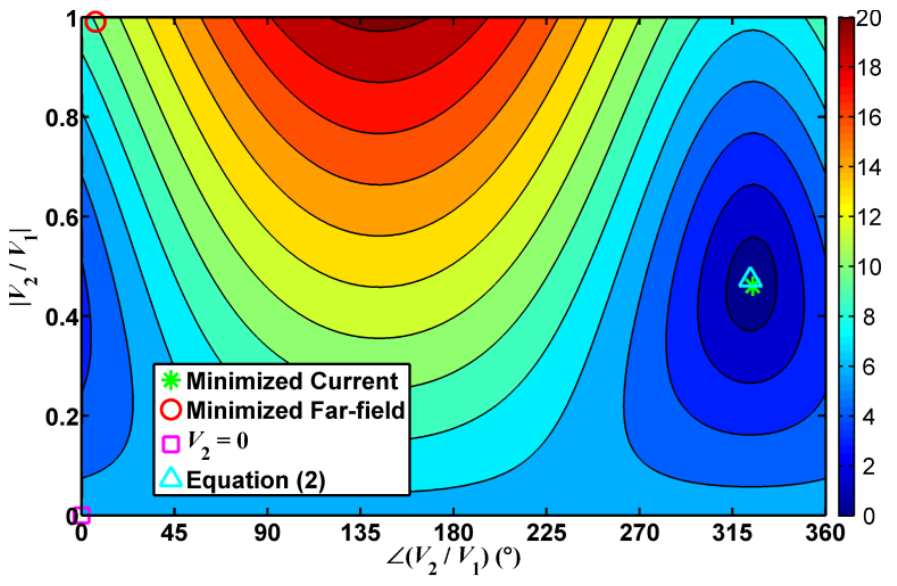

(a)

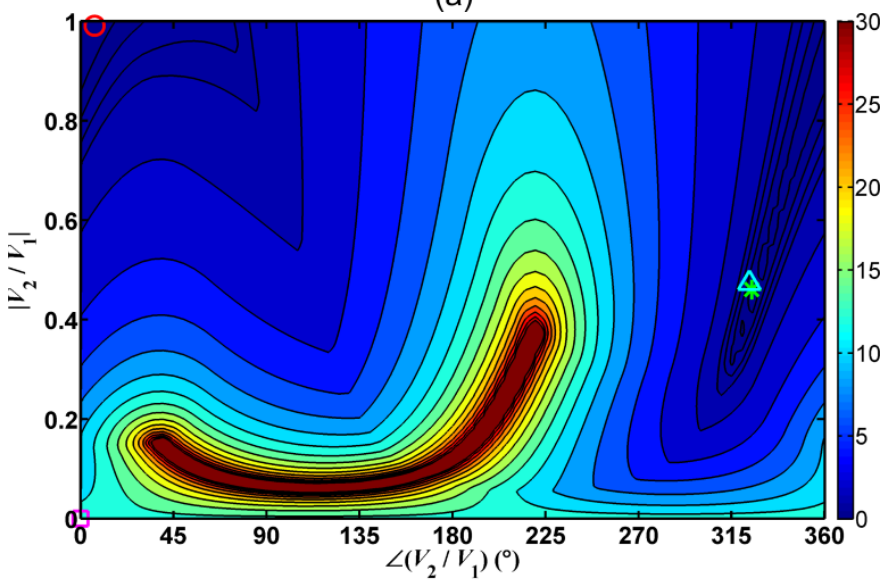

(b)

Fig. 3. (a) The maximum of the magnitude of the current (mA) on Dipole 2 and (b) the maximum gain deviation $(\mathrm{dB})$ from the peak gain in the H-plane for the two-element dipole array shown for variations in voltage source $V_{2}$ with $f_{1}=2.5763 \mathrm{GHz}$. Dipole 1 is excited with $V_{1}=1 \angle 0^{\circ} \mathrm{V}$. Superimposed (marker symbols) are the minima of the contour plots, $V_{2}=0 \mathrm{~V}$, and the voltage obtained from (2), as summarized in Table I.

TABLE I

VOLTAGE EXCITATIONS FOR THE TWO-DiPOLE ARRAY

\begin{tabular}{lcc}
\hline \hline \multirow{3}{*}{ Condition } & $f_{1}=2.576 \mathrm{GHz}$, & $f_{2}=3.040 \mathrm{GHz}$, \\
& $V_{1}=1 \angle 0^{\circ}$ & $V_{2}=1 \angle 0^{\circ}$ \\
$V_{2}(\mathrm{~V})$ & $V_{1}(\mathrm{~V})$ \\
\hline Minimized current & $0.460 \angle 324.6^{\circ}$ & $0.682 \angle 323.1^{\circ}$ \\
Minimized pattern variation & $0.990 \angle 6.9^{\circ}$ & $0.546 \angle 326.4^{\circ}$ \\
Short circuited dipole & 0 & 0 \\
Equation (2) & $0.473 \angle 323.5^{\circ}$ & $0.988 \angle 321.1^{\circ}$ \\
\hline \hline
\end{tabular}

In Fig. 4(a) a non-zero current distribution is observed on both dipoles for all cases (including when (2) is used to determine the excitation). The voltage given by (2) is close to the minimum of Fig. 3(a), i.e. the minimized current, but very different from the case with minimized gain deviation. The results show that for a pure omni-directional pattern to be attained a larger current is required on Dipole 2 than on Dipole 1 with a $V_{2}$ similar to that of $V_{1}$. It may be noted here that, for this specific case where, at the operating frequency, the length of Dipole 2 is close to a half-wavelength and the induced current is thus expected to be dominated by the fundamental mode, the results obtained by using (2) or by minimizing the dipole current produce near-perfect omni-directional patterns. 
This is not the case at the higher resonant frequency, or where more dipoles are considered - as will be shown in the following paragraphs. For the case that $V_{2}=0$ the non-excited dipole acts as a director as is the case for Yagi-Uda antennas [5].
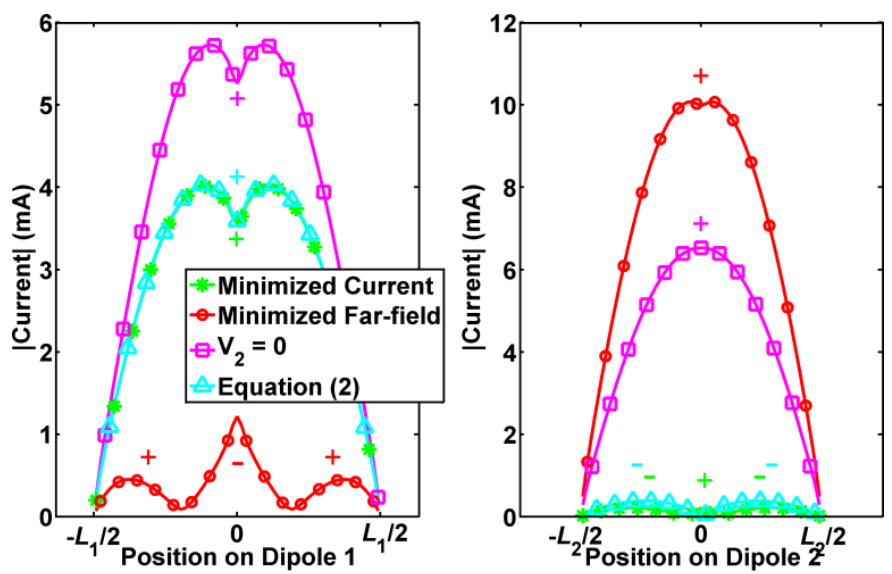

(a)

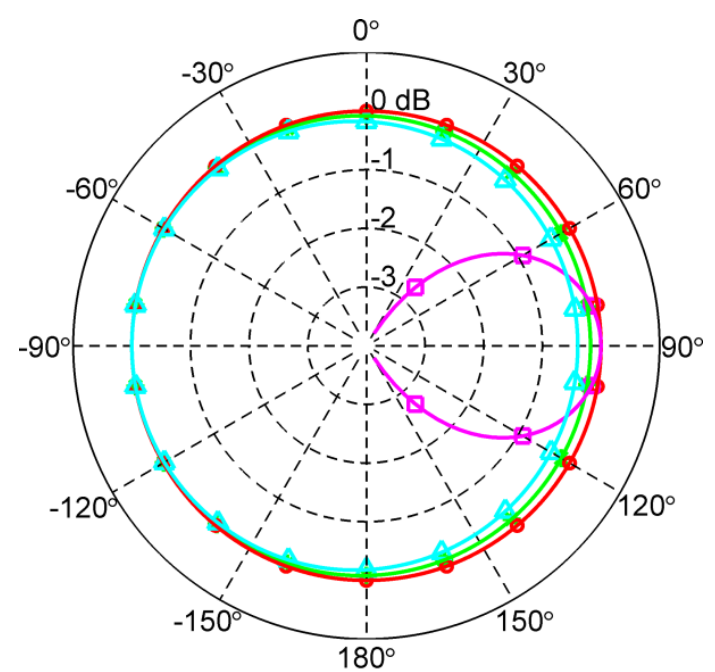

(b)

Fig. 4. (a) The magnitude of the current distribution on the dipoles and (b) the $\mathrm{H}$-plane radiation pattern cuts of the two-element dipole array excited as in Table I at $2.5763 \mathrm{GHz}$. The positive and negative symbols in (a) indicate the phase of the current. Note that (2) produces a near omni-directional pattern for this case where the terminal current vanishes on the shorter dipole.

The simulations were repeated at the higher frequency $f_{2}$, i.e. $f_{2}$ equal to $3.04 \mathrm{GHz}$. In this case $V_{2}=1 \angle 0^{\circ} \mathrm{V}$ and $V_{1}$ is the parameter that is investigated. The results are given in Fig. 5 and Fig. 6 and the voltages are summarized in Table I. Again the minima of the contour plots occur at different voltages and the result from (2) does not coincide with either of these minima. It is observed in Fig. 6(a) that the voltage given by (2) does force the terminal current to zero; however, the current distribution along the length of the dipole is quite large, and indeed resembles a higher order current mode. This results in an array radiation pattern that is considerably different from an omni-directional radiation pattern as shown in Fig. 6(b). For the case that the current distribution is minimized the pattern becomes more omni-directional, but in order to get a perfect omni-directional pattern (gain deviation of less than $0.006 \mathrm{~dB}$ ) the current distribution needs to have a particular profile that can be determined by optimization of the voltage $V_{1}$. Again, non-zero currents are observed on both dipoles for all cases.

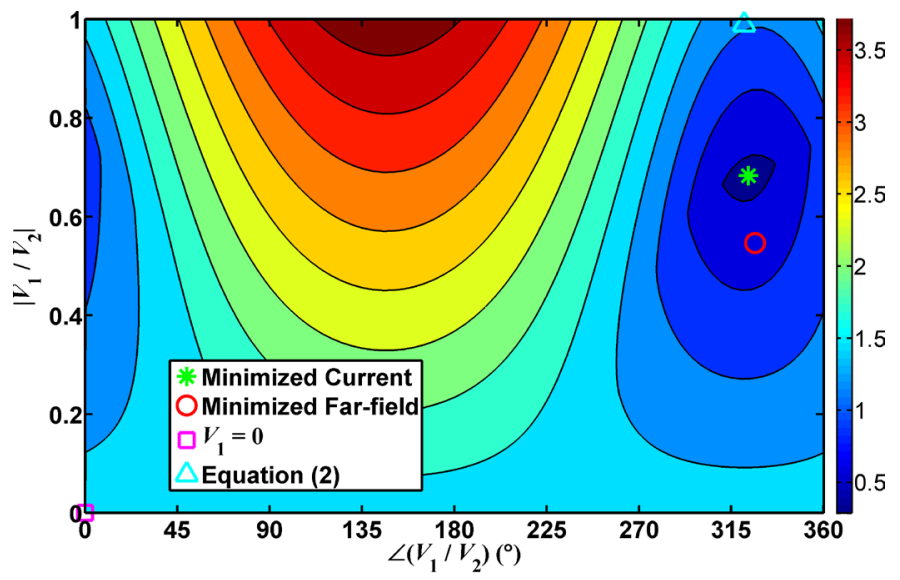

(a)

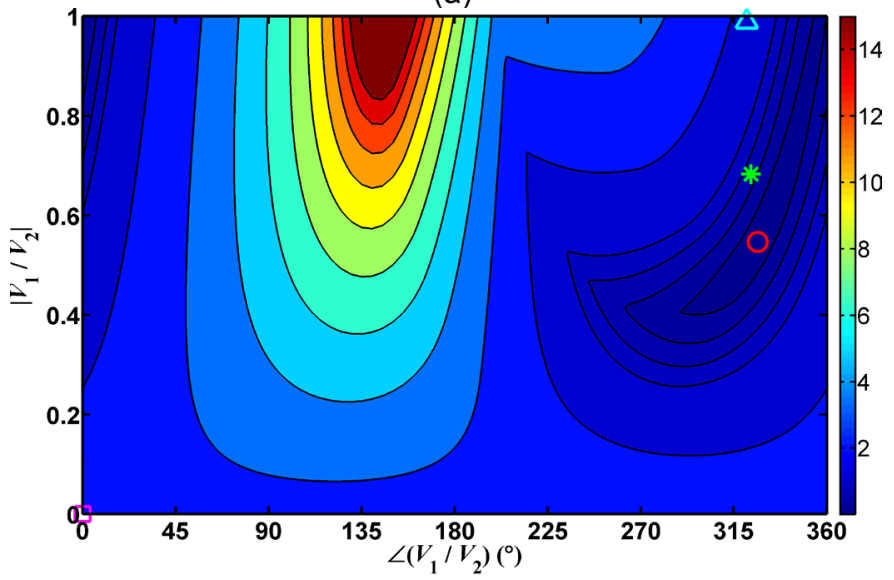

(b)

Fig. 5. (a) The maximum of the magnitude of the current (mA) on Dipole 1 and (b) the maximum gain deviation $(\mathrm{dB})$ from the peak gain in the H-plane for the two-element dipole array shown for variations in voltage source $V_{1}$ with $f_{2}=3.04 \mathrm{GHz}$. Dipole 2 is excited with $V_{2}=1 \angle 0^{\circ} \mathrm{V}$. Superimposed (marker symbols) are the minima of the contour plots, $V_{1}=0 \mathrm{~V}$, and the voltage obtained from (2), as summarized in Table I.

Physically disconnecting the dipole arms by removing the port segment in FEKO produces exactly the same radiation pattern as was obtained using (2).

When coupling to a dipole which is in the order of a half wavelength long, forcing the terminal current to zero will lead to a small total current since mainly the fundamental mode is excited. However, in general, when coupling to electrically longer dipoles, where several current modes may exist, forcing the terminal currents to zero will not imply small total current. We thus conclude that the voltage given by (2) does not lead to a condition of constant radiation characteristics. However, constant radiation characteristics may be achievable with the correct choice of the voltage excitations. The radiation characteristics are dependent on the current distribution on each dipole and these can be changed by the applied voltage excitations. In general, the voltage excitations cannot force the 
current distribution along the entire length of the dipole to zero as the distributions from the applied voltage and that from coupling are different.
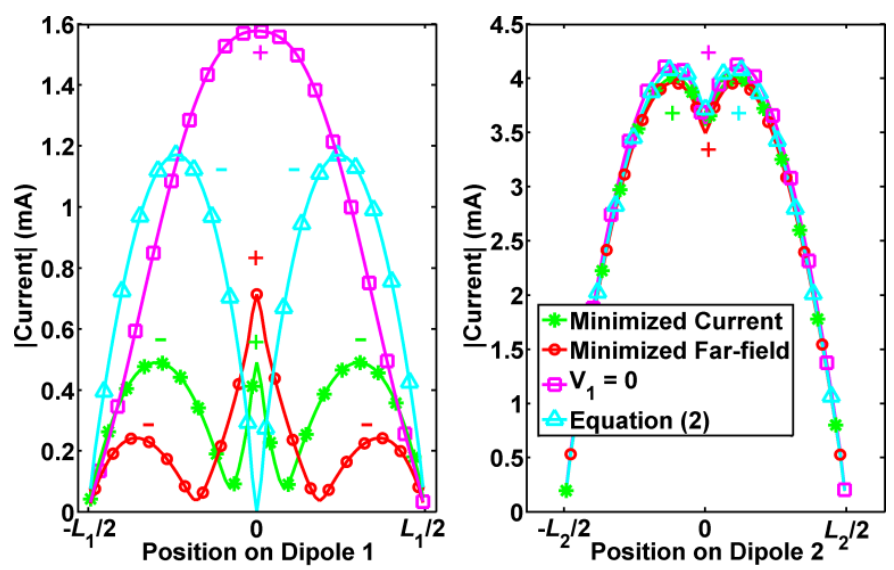

(a)

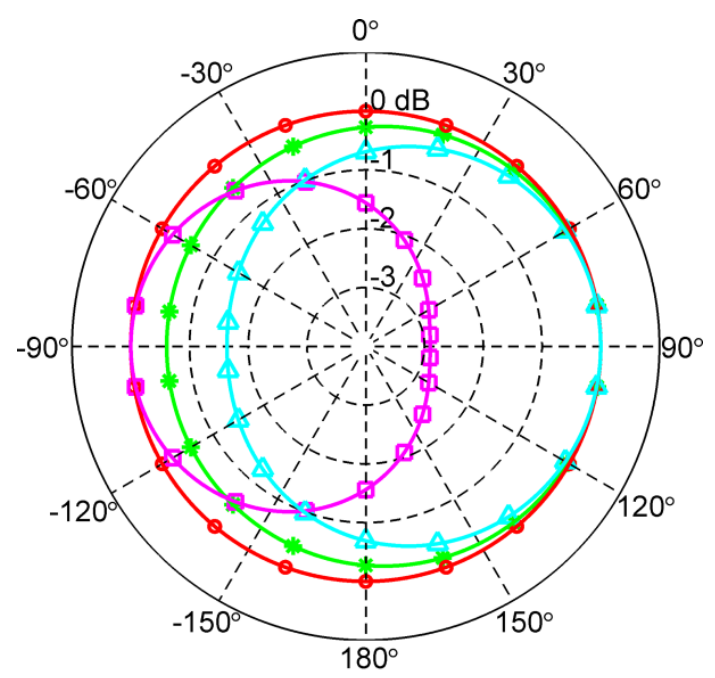

(b)

Fig. 6. (a) The magnitude of the current distribution on the dipoles and (b) the $\mathrm{H}$-plane radiation pattern cuts of the two-element dipole array excited as in Table I at $3.04 \mathrm{GHz}$. The positive and negative symbols in (a) indicate the phase of the current. Note that (2) does not produce an omni-directional pattern for this case where the terminal current vanishes on the longer dipole.

\section{FIVE-ElEMENT LoG-PERIODIC Dipole ARRAY}

Using the same dimensions as in [3] for a five-element LPDA we have analyzed the array using FEKO with the terminal excitations determined from (2). The resultant $\mathrm{H}$-plane radiation pattern cuts are shown in Fig. 7. At $3.04 \mathrm{GHz}$ the array has an almost omni-directional radiation pattern and this pattern is similar to the one given in [3]. Slight deviations are due to the exact modeling of the port structure. In [3] the result was only given for the $3.04 \mathrm{GHz}$ case and opportunely not at the other resonant frequencies. It is clear from Fig. 7 that at the higher frequencies the patterns are no longer omni-directional.

The correct terminal voltages can be obtained by means of a global optimization to achieve omni-directional radiation patterns at the five resonant frequencies.

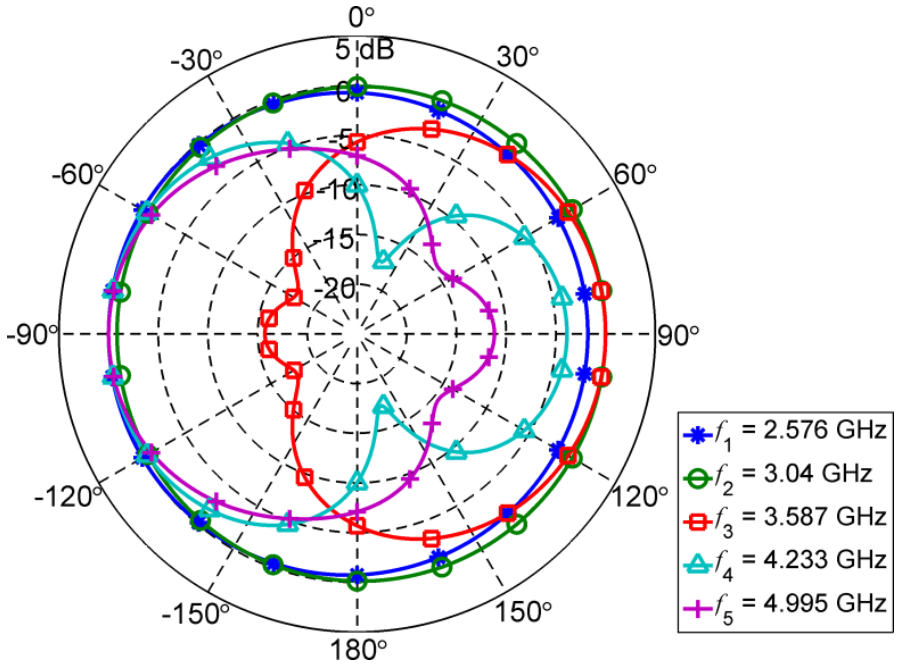

Fig. 7. The H-plane radiation pattern cuts of the five-element dipole array at the dipole's resonant frequencies using the voltage excitations from (2). Open circuiting the non-resonant element ports for each excitation produces exactly the same results.

\section{CONCLUSION}

Equivalent circuit model representations of an antenna element can provide accurate models for the currents and voltages at the terminal. However, they do not, in general, present an accurate model for the currents on the antenna structure that produces electromagnetic radiation - especially when the element becomes electrically large. Constant radiation characteristics appear to be achievable for a log-periodic array with the correct choice of terminal voltages. These terminal voltages cannot be obtained from an analytic formula as given in [3], but need to be determined by a global search algorithm.

\section{REFERENCES}

[1] R. H. DuHamel and D. E. Isbell, "Broadband logarithmically periodic antenna structures," IRE National Convention Record, pt. 1, pp. 119-128, 1957.

[2] C. A. Balanis, Antenna Theory, 3rd ed. Hoboken, NJ, USA: John Wiley Sons, 2005, ch. 4 and 8.

[3] J. Yang, "On conditions for constant radiation characteristics for log-periodic array antennas," IEEE Trans. Antennas Propagat., vol. 58, no. 5, pp. 1521-1526, May 2010.

[4] EM Software \& Systems - S.A. (Pty) Ltd, Stellenbosch, South Africa, FEKO, Suite 6.2. Available: http://www.feko.info.

[5] S. Uda and Y. Mushiake, Yagi-Uda antenna, Sasaki Printing and Publishing Company, Ltd., Sendai, Japan, 1954. 